\title{
A nearly cylindrically symmetric source in Brans-Dicke gravity as the generator of the rotational curves of the galaxies
}

\author{
S. Mittmann dos Santos ${ }^{1,2, a}$, J. M. Hoff da Silva ${ }^{1, b}$, M. E. X. Guimarães ${ }^{3, c}$, J. L. Neto ${ }^{4, d}$ \\ ${ }^{1}$ Universidade Estadual Paulista Júlio de Mesquita Filho-UNESP, Campus de Guaratinguetá, Guaratinguetá, SP 12516-410, Brazil \\ ${ }^{2}$ Instituto Federal de Educação, Ciência e Tecnologia do Rio Grande do Sul-IFRS, Campus Porto Alegre, Porto Alegre, RS 90030-041, Brazil \\ ${ }^{3}$ Instituto de Física, Universidade Federal Fluminense-UFF, Av. Gal. Milton Tavares de Souza, s/n, Niterói, RJ 24210-346, Brazil \\ ${ }^{4}$ Instituto de Física, Universidade Federal do Rio de Janeiro-UFRJ, Av. Athos da Silveira Ramos, 149, Cidade Universitária, Rio de Janeiro, \\ RJ 21941-909, Brazil
}

Received: 28 August 2017 / Accepted: 20 November 2017 / Published online: 8 December 2017

(C) The Author(s) 2017. This article is an open access publication

\begin{abstract}
Observation shows that the velocities of stars grow by approximately $2-3$ orders of magnitude when the distances from the centers of the galaxies are in the range of $0.5-82.3 \mathrm{kpc}$, before they begin to tend to a constant value. Up to now, the reason for this behavior is still a matter for debate. In this work, we propose a model which adequately describes this unusual behavior using a (nearly) cylindrical symmetrical solution in the framework of a scalar-tensor-like (the Brans-Dicke model) theory of gravity.
\end{abstract}

\section{Introduction}

Brans-Dicke (BD) gravitation [1] describes gravitational phenomena with the aid of a scalar field $\tilde{\phi}$ in addiction to the usual tensorial field in General Relativity (GR). The gravitational action of the $\mathrm{BD}$ theory, which gives rise to the dynamic equations, is given by (in the Jordan-Fierz physical frame) ${ }^{1}$

$S=\frac{1}{16 \pi} \int d^{4} x \sqrt{-\tilde{g}}\left(\tilde{\phi} \tilde{R}-\frac{\omega}{\tilde{\phi}} \partial^{\mu} \tilde{\phi} \partial_{\mu} \tilde{\phi}\right)+S_{\text {matter }}$.

The dimensionless parameter $\omega$, called the BD coupling parameter, determines the deviation of the results obtained in this theory from those in GR. The lower the value of $\omega$, the more different are the correspondent results. For instance, for the Solar System, where GR does extremely \footnotetext{
the Jordan-Fierz frame.

ae-mail: sergio.santos@poa.ifrs.edu.br

be-mail: hoff@feg.unesp.br

ce-mail: emilia@if.uff.br

de-mail: jlneto@if.ufrj.br
}

${ }^{1}$ We have reserved the terms with tilde for the evaluated quantities in well, $|\omega|>40,000[2,3]$. Thus, one could think of discarding a scalar-tensor-like theory. Nevertheless, during the investigation of the cosmological evolution of the Universe with a scalar-tensorial theory it was concluded that GR is an attractor of the scalar-tensor theory, because the scalar field dynamics would have been gradually suppressed [47]. Therefore, a scalar-tensor gravitation theory seems to be most appropriate for describing sources that originated shortly after the Big Bang, when scalar field strength and its variation were still important.

It is now known that only less than $5 \%$ of what makes up the Universe is well understood, consisting of baryonic matter and radiation. Dark matter and dark energy, which dominate our Universe, have not yet their origin and evolution satisfactorily explained. Dark matter, supposedly present in the halo region of galaxies, seems to be the mechanism that causes the unusual behavior of the tangential velocity of the stars which is superior to that predicted by interaction with visible matter [8-12]. Moreover, in addition to the velocity larger than expected, as the distance of the stars from the center of the galaxy grows, their velocities tend to a constant value [10]. An alternative explanation to that behavior is to consider a scalar-tensor theory which might solve the rotational curves paradigm [13-15]. The results of the above-mentioned references do reproduce the large distance behavior of the velocity with the expected order of magnitude. However, they give no information on how the intensity of that velocity evolves.

The stars of the galaxies studied in Ref. [10] are distributed along an approximately flat region. Therefore, it is possible to suppose that a symmetrically cylindrical spacetime could describe the rotation curves. Symmetrically cylindrical sources are widely studied in several areas. Among these sources, some prominence can be given to the spinning 
cosmic strings, which would have originated from a spontaneous symmetry breaking, occurring from a phase transition of the primordial Universe. They have an angular velocity about the longitudinal axis of symmetry and, being straight strings, they preserve the Lorentz invariance along its symmetry axis. Many studies in the GR theory indicate that there are closed timelike curves (CTCs) at least in part of the spacetime around these strings [16]. As they would have formed in the primordial Universe, a recent work has demonstrated what occurs when, instead of GR, the description of these defects is analyzed in the framework a BD gravity [17]. As in GR, the results indicated that CTCs are also present in the spacetime around these strings. There is a great deal of discussion as to whether CTCs exist or not in the Universe, since their confirmation would violate the causality principle. It is argued that some unknown form of matter could allow the existence of CTCs [18], which cannot be ruled out, since we do not have a full understanding of what most of the Universe is made up. References [17,19] analyzed the energy quantization of a particle with a non-inertial motion around a straight spinning cosmic string and concluded that it would be unstable, because it would have an angular velocity much higher than would be physically acceptable. Thus, if this type of string would have formed in the primordial Universe, it should not have survived.

The main goal of this paper is to present the full solution for a nearly cylindrically symmetric spacetime in the BD gravity and use it, as an alternative to dark matter, to describe the rotational curves of the galaxies in Ref. [10]. We show the solutions for the equations of motion for the action (1) when Lorentz invariance along the $z$ longitudinal axis of symmetry is slightly violated, given that a straight source may be unstable, as happens with the spinning cosmic strings. The relevant invariance when dealing with the gravitational phenomena is to be taken with respect to general coordinate transformations. Since we are interested in the vacuum solution, less importance is assigned to the source of this spacetime. Nevertheless, one may bear in mind a (nonstraight) spinning cosmic string with a very large radius. If one thinks of the source as a Lorentz violating object, then it is important to remember that in many references it has been pointed out that the search for a theory of grand unification leads to a spontaneous breaking of Lorentz symmetry at the time of the primordial Universe [20-23], when we consider that the formation of the source has occurred and, therefore, also the BD gravity was dominant over GR. Moreover, in order not to lose the (nearly) cylindrical symmetry adopted, the ratio between the $\mathrm{d} t^{2}$ and $\mathrm{d} z^{2}$ coefficients of the line element is approximately unity.

We rescale the equations of motion obtained from the action in the Jordan-Fierz frame (1) to the Einstein (or conformal) frame in which the scalar and the tensor degrees of freedom do not mix. This makes the interpretation and han- dling of the solutions easier. It is important to strength that the qualitative results obtained in one frame are equivalent to those in the other. In any case, throughout the paper the interpretation of the results will be performed for the two frames, since both cases may be important in the description of a given gravitational system [24,25]. With the solutions of the dynamic equations at hand, we verified that the spacetime is indeed regular as evidenced by the analysis of the Kretschmann scalars. Depending on the values adopted for the integration constants, CTCs may appear or not in certain regions. The resulting spacetime adequately reproduces the motion of the stars of the galaxies studied in Ref. [10], either in the regions where CTCs exist or where the metric is Euclidean. This is the main result of our paper. We also provide additional argumentation in favor of using BD gravity to address this problem.

This paper is organized as follows. In Sect. 2, we first present the dynamic equations for the $\mathrm{BD}$ action in the Jordan-Fierz and Einstein frames. From the equations in the Einstein frame, the exact solution for a nearly cylindrically symmetric spacetime is obtained. In Sect. 3, the physical properties of the spacetime obtained in the previous section are described. In Sect. 4, we show that the resulting spacetime adequately describes the rotational curves of Sc type galaxies. In Sect. 5, it is discussed that BD gravitation is essential for the aforementioned application. In Sect. 6, we summarize our findings and discuss the relevant conclusions. We leave for the appendix complete tables encompassing relevant data and calculation results of the presented analysis.

\section{Solutions for a nearly cylindrically symmetric spacetime in Brans-Dicke gravity}

The equations of motion from the action (1) in the JordanFierz frame are

$$
\begin{aligned}
\tilde{G}_{\mu \nu}= & \frac{\omega}{\tilde{\phi}^{2}}\left(\partial_{\mu} \tilde{\phi} \partial_{\nu} \tilde{\phi}-\frac{1}{2} \tilde{g}_{\mu \nu} \tilde{g}^{\rho \sigma} \partial_{\rho} \tilde{\phi} \partial_{\sigma} \tilde{\phi}\right) \\
& +\frac{1}{\tilde{\phi}}\left(\partial_{\mu} \partial_{\nu} \tilde{\phi}-\tilde{g}_{\mu \nu} \tilde{\square} \tilde{\phi}\right)+\frac{8 \pi}{\tilde{\phi}} T_{\mu \nu}, \\
\tilde{\square} \tilde{\phi}= & \frac{1}{\sqrt{-\tilde{g}}} \partial_{\mu}\left(\sqrt{-\tilde{g}} \partial^{\mu} \tilde{\phi}\right)=\frac{8 \pi}{2 \omega+3} T .
\end{aligned}
$$

There is a more convenient way to handle the $\mathrm{BD}$ equations which is through the Einstein frame. In this frame, $\tilde{g}_{\mu \nu}$ and $\tilde{\phi}$ from Eqs. (2) are rescaling by the new dynamical variables $g_{\mu \nu}$ and $\phi$, as in [26]:

$$
\begin{aligned}
\tilde{g}_{\mu \nu} & =\exp (2 \kappa \phi) g_{\mu \nu}, \\
\tilde{\phi} & =\frac{1}{G} \exp (-2 \kappa \phi),
\end{aligned}
$$


where

$\kappa^{2}=\frac{1}{2 \omega+3}$

and $G$ is the effective gravitational Newtonian constant. Thus, in the Einstein frame, Eqs. (2) can be written as

$$
\begin{aligned}
G_{\mu \nu} & =2 \partial_{\mu} \phi \partial_{\nu} \phi-g_{\mu \nu} g^{\rho \sigma} \partial_{\rho} \phi \partial_{\sigma} \phi+\frac{8 \pi}{\phi} T_{\mu \nu}, \\
\square \phi & =\frac{8 \pi}{2 \omega+3} T .
\end{aligned}
$$

For the system we are about to consider, since the qualitative behavior is basically the same in Jordan-Fierz and Einstein frames, we shall present and interpret the results in the manageable Einstein frame and only occasionally make reference to the Jordan-Fierz frame.

The most general cylindrically symmetric line element to be addressed in this work is [16]

$\mathrm{d} s^{2}=-\left(\mathrm{e}^{\alpha} \mathrm{d} t+M \mathrm{~d} \varphi\right)^{2}+r^{2} \mathrm{e}^{-2 \alpha} \mathrm{d} \varphi^{2}+\mathrm{e}^{2(\beta-\alpha)}\left(\mathrm{d} r^{2}+\mathrm{d} z^{2}\right)$,

where the angular coordinate varies, in principle, in the range $0 \leq \varphi<2 \pi$. By means of the cylindrical symmetry we have $\alpha, \beta$ and $M$ as functions of the, strictly positive, radial distance $r$ only. In order not to completely give up cylindrical symmetry we adopt $\frac{\beta}{\alpha} \approx 2$. It is in this sense that we have nearly cylindrical symmetry. Notice that an eventual source for this spacetime would not be straight and slightly breaking Lorentz invariance. The field equations were obtained in detail previously [17]. Therefore we will suppress the calculations. They read, for the vacuum case,

$$
\begin{aligned}
\mathcal{G}^{t}{ }_{t}: & -3 \Omega^{2}-\left(2 \alpha^{\prime \prime}-\beta^{\prime \prime}+\frac{2 \alpha^{\prime}}{r}-\alpha^{\prime 2}\right) \mathrm{e}^{2(\alpha-\beta)} \\
& =-\mathrm{e}^{2(\alpha-\beta)} \phi^{\prime 2} \\
\mathcal{G}^{r} & : \Omega^{2}+\left(\frac{\beta^{\prime}}{r}-\alpha^{\prime 2}\right) \mathrm{e}^{2(\alpha-\beta)}=\mathrm{e}^{2(\alpha-\beta)} \phi^{\prime 2} \\
\mathcal{G}^{z} & :-\Omega^{2}+\left(\alpha^{\prime 2}-\frac{\beta^{\prime}}{r}\right) \mathrm{e}^{2(\alpha-\beta)}=-\mathrm{e}^{2(\alpha-\beta)} \phi^{\prime 2} \\
\mathcal{G}_{\varphi}^{\varphi} & : \Omega^{2}+\left(\beta^{\prime \prime}+\alpha^{\prime 2}\right) \mathrm{e}^{2(\alpha-\beta)}=-\mathrm{e}^{2(\alpha-\beta)} \phi^{\prime 2} \\
\mathcal{G}_{\varphi}^{t}: & {\left[\Omega^{\prime}+2 \alpha^{\prime} \Omega+\Omega\left(\beta^{\prime}-\alpha^{\prime}\right)\right] \mathrm{e}^{\alpha-\beta}=0 } \\
\square \phi & : \frac{\phi^{\prime}}{2} \frac{\mathrm{d}}{\mathrm{d} r}\left\{\ln \left[\mathrm{e}^{4(\beta-\alpha)} r^{2}\left(\mathrm{e}^{2(\alpha-\beta)} \phi^{\prime}\right)^{2}\right]\right\} \mathrm{e}^{2(\alpha-\beta)}=0,
\end{aligned}
$$

where

$\Omega=\frac{M \alpha^{\prime}-M^{\prime}}{2 r} e^{2 \alpha-\beta}$.
The prime $\left({ }^{\prime}\right)$ in the above equations refers to a derivative with respect to $r$.

Equations (11) and (10) are equal and Eq. (14) has the following solution for the scalar field:

$\phi(r)=C_{2}+C_{1} \ln r$,

where $C_{1}$ and $C_{2}$ are constants. Adding Eqs. (9) and (10) and using our definition of $\Omega$ (15) we get

$\mathrm{e}^{2 \alpha}\left(M^{\prime}-M \alpha^{\prime}\right)^{2}=-2 r\left(2 \alpha^{\prime}-\beta^{\prime}+2 r \alpha^{\prime \prime}-r \beta^{\prime \prime}\right)$.

Subtracting Eqs. (9) from (12) and substituting the term $\mathrm{e}^{2 \alpha}\left(M^{\prime}-M \alpha^{\prime}\right)^{2}$ by the right-hand side of Eq. (17), we have

$\alpha^{\prime}-\beta^{\prime}+r \alpha^{\prime \prime}-r \beta^{\prime \prime}=0$.

If $\alpha-\beta=\gamma$, Eq. (18) can be rewritten as

$\gamma^{\prime}+r \gamma^{\prime \prime}=0$

which has the solution

$\gamma=C_{b}+C_{a} \ln r$,

where $C_{a}$ and $C_{b}$ are constants. Thus,

$\beta(r)=\alpha(r)-\left(C_{b}+C_{a} \ln r\right)$.

Equation (12) can be rewritten as

$\frac{1}{4}\left[\frac{\mathrm{e}^{2 \alpha}\left(M^{\prime}-M \alpha^{\prime}\right)^{2}}{r^{2}}+4\left(\alpha^{\prime 2}+\beta^{\prime \prime}\right)\right]=-\phi^{\prime 2}$.

Substituting the term $\mathrm{e}^{2 \alpha}\left(M^{\prime}-M \alpha^{\prime}\right)^{2}$ from Eq. (22) by the right-hand side of Eq. (17), we have

$\phi^{\prime 2}=\frac{2 \alpha^{\prime}-2 r \alpha^{\prime 2}-\beta^{\prime}+2 r \alpha^{\prime \prime}-3 r \beta^{\prime \prime}}{2 r}$.

When we use Eqs. (16) and (21) in Eq. (23), the solution for $\alpha$ is

$\alpha=C_{4}+\frac{1}{2}\left[\ln r-k_{1} \ln r+\ln \left(C_{3}+r^{2 k_{1}}\right)\right]$,

where $C_{3}, C_{4}$ and $k_{1}$ are constants, with

$k_{1}=\sqrt{1-4 C_{1}^{2}-4 C_{a}} \geq 0$.

Now, when we use the solutions (16), (21) and (24) in Eq. (17), the solutions for $M$ are 


$$
\begin{aligned}
M(r)_{ \pm}= & C_{5} \sqrt{r^{1-k_{1}}\left(C_{3}+r^{2 k_{1}}\right)} \\
& \pm \sqrt{-\frac{r^{1+3 k_{1}}}{C_{3} \mathrm{e}^{2 C_{4}}\left(C_{3}+r^{2 k_{1}}\right)}}
\end{aligned}
$$

where $C_{5}$ is a constant. Summarizing, the exact solutions for the system (9)-(14) read

$$
\begin{aligned}
\phi(r) & =C_{2}+C_{1} \ln r \\
\beta(r) & =\alpha(r)-\left(C_{b}+C_{a} \ln r\right), \\
\alpha(r) & =C_{4}+\frac{1}{2}\left[\ln r-k_{1} \ln r+\ln \left(C_{3}+r^{2 k_{1}}\right)\right], \\
M(r)_{ \pm} & =C_{5} \sqrt{r^{1-k_{1}}\left(C_{3}+r^{2 k_{1}}\right)} \\
& \pm \sqrt{-\frac{r^{1+3 k_{1}}}{C_{3} \mathrm{e}^{2 C_{4}}\left(C_{3}+r^{2 k_{1}}\right)}}
\end{aligned}
$$

where $r>0, \frac{\beta(r)}{\alpha(r)}=C_{\alpha \beta} \approx 2$ (e.g., the nearly cylindrical symmetry condition) and, for the solutions to be real, it is necessary that

$$
\begin{aligned}
C_{3} & <0, \\
\left|C_{3}\right| & <r^{2 k_{1}} .
\end{aligned}
$$

According to Eqs. (27) and (28), $C_{3}$ can be written as

$$
C_{3}=-a_{C_{3}} r_{\min }^{2 k_{1}}
$$

where $0<a_{C_{3}}<1$ and $r_{\min }=\min r$, a value for the radial coordinate below which the system cannot be associated to the vacuum.

A final remark on recovering GR out from this scalartensorial formalism: as we are working with vacuum dynamical equations we cannot implement the condition $\omega \rightarrow \infty$ in order to keep track of the GR limit. However, it is possible equivalently to suppress the scalar field dynamics taking $C_{1}=0$. Henceforward, we shall be making contact with GR by means of this $\left(C_{1}=0\right)$ necessary condition.

\section{Spacetime properties}

From the line element (8), with the solutions (21), (24) and (26), the Kretschmann scalars $R^{2}, R_{\mu \nu} R^{\mu \nu}, R_{\alpha \beta \mu \nu} R^{\alpha \beta \mu \nu}$ and $C_{\alpha \beta \mu \nu} C^{\alpha \beta \mu \nu}$, where $C_{\alpha \beta \mu \nu}$ stands for the Weyl tensor, in the Einstein frame, are functions of $\alpha, \beta, M$ and their first and second derivatives with respect to $r$. In the JordanFierz frame, the line element is rewritten as (3) and, with the same solutions (21), (24) and (26) plus the solution (16), the Kretschmann scalars are functions of $\alpha, \beta, M, \phi$ and their first and second derivatives with respect to $r$, and of the parameter $\omega$. In the two frames, the results show that the spacetime is well behaved for $r>0$.

When the $\varphi$ coefficient of the line element (8) is negative, CTCs appear. For this to happen, it is necessary that the following inequality holds:

$g_{\varphi \varphi}<0$.

Then, considering that

$g_{\varphi \varphi}=\mathrm{e}^{-2 \alpha} r^{2}-M^{2}$

and with the choice of certain constants for the solutions (24) and (26), it is possible to determine the intervals for the coordinate $r$ where CTCs appear. For a specific example and considering a dimensional parameters, when $C_{4}=0.1$, $C_{5}=1, C_{a}=0.08, r_{\min }=1$ and the $M$ function is taken with the negative sign in the second term of Eq. (26), i.e., $M=M_{-}$, Fig. 1 shows the behavior of $g_{\varphi \varphi}$ for distinct $a_{C_{3}}$ and $C_{1}$ values. As already remarked in Sect. 2 , if $C_{1}=0$ the scalar field $\phi$ is constant and the theory approaches GR.

Thus, we can observe in Fig. 1 that, for the same $a_{C_{3}}$, the region where there is no CTC (when $g_{\varphi \varphi}>0$ ) is larger when we are in the context of BD theory. This reasoning does not apply to $a_{C_{3}}=0.1$ because, in this case, there is CTC in both theories for any value of the coordinate $r$. For $a_{C_{3}}=0.9$, there is no CTC up until to the region between $r=10^{6}$ and $r=10^{7}$, for the BD gravitation, and between $r=10^{1}$ and $r=10^{2}$, for the GR gravitation. When $a_{C_{3}}=0.5$, in $\mathrm{BD}$, that region is between $r=10^{2}$ and $r=10^{3}$, whereas, in GR, that region is between $r=10^{0}$ and $r=10^{1}$. The fact that the region where there are CTCs, defined from a certain distance from the $z$-axis (and not up to a certain distance, as with the straight spinning cosmic strings $[16,17]$ ) resembles what is predicted in the Goedel solution, when the CTCs appear when the source distance is from a so-called critical radius [27].

\section{Rotational curves in galaxies}

The Lagrangian of a particle in the spacetime described by the metric (8) is given by [28]

$$
\begin{aligned}
2 \mathcal{L}= & -\mathrm{e}^{2 \alpha} \dot{t}^{2}-M^{2} \dot{\varphi}^{2}-2 \mathrm{e}^{\alpha} M \dot{t} \dot{\varphi}+r^{2} \mathrm{e}^{-2 \alpha} \dot{\varphi}^{2} \\
& +\mathrm{e}^{2(\beta-\alpha)} \dot{r}^{2}+\mathrm{e}^{2(\beta-\alpha)} \dot{z}^{2} .
\end{aligned}
$$

The canonical momenta associated with the Lagrangian (32) are

$$
\begin{aligned}
& p_{t}=\frac{\partial \mathcal{L}}{\partial \dot{t}}=-E, \\
& p_{\varphi}=\frac{\partial \mathcal{L}}{\partial \dot{\varphi}}=L,
\end{aligned}
$$




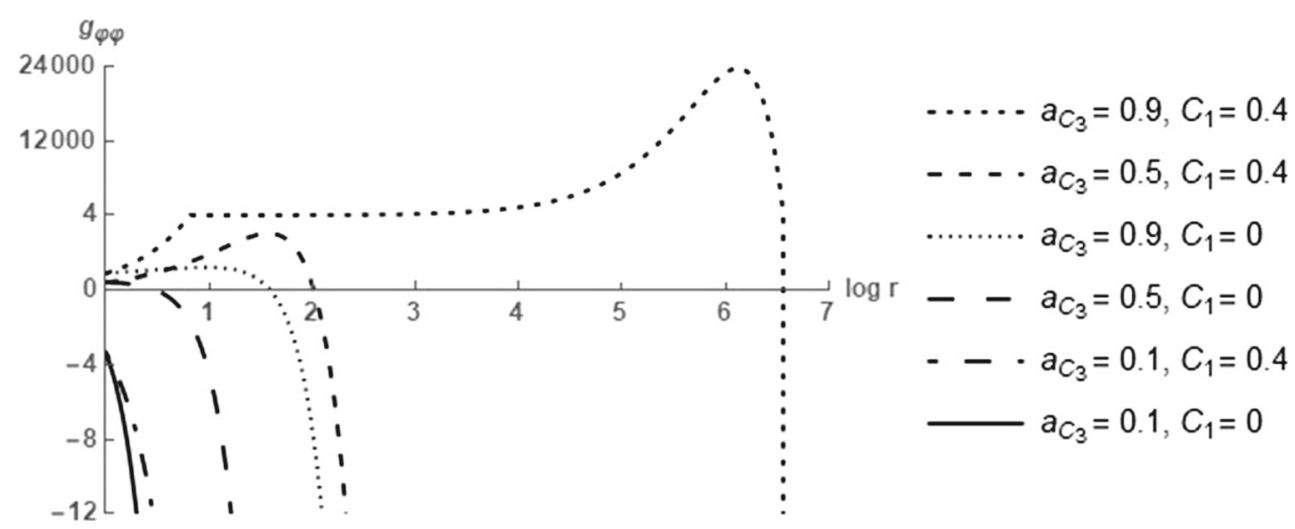

Fig. 1 Behavior of $g_{\varphi \varphi}$ for distinct $a_{C_{3}}$ and $C_{1}$ values, with $C_{4}=0.1, C_{5}=1, C_{a}=0.08, r_{\min }=1$ and $M=M_{-}$. When $g_{\varphi \varphi}>4$ the adopted scale for the vertical axis is different from that when $g_{\varphi \varphi} \leq 4$

$p_{r}=\frac{\partial \mathcal{L}}{\partial \dot{r}}$,

$p_{z}=\frac{\partial \mathcal{L}}{\partial \dot{z}}$,

where $E$ and $L$ are conserved quantities [28]. By Eqs. (33) and (34), we have

$\dot{t}=-\frac{\mathrm{e}^{-2 \alpha}\left(-E r^{2}+L \mathrm{e}^{3 \alpha} M+E \mathrm{e}^{2 \alpha} M^{2}\right)}{r^{2}}$,

$\dot{\varphi}=\frac{\mathrm{e}^{\alpha}\left(L \mathrm{e}^{\alpha}+E M\right)}{r^{2}}$.

The Hamiltonian of this particle is

$\mathcal{H}=p_{\mu} \dot{x}^{\mu}-\mathcal{L}=p_{t} \dot{t}+p_{\varphi} \dot{\varphi}+p_{r} \dot{r}+p_{z} \dot{z}-\mathcal{L}$.

Let us consider the stars as truly test particles moving in a circular motion in the equatorial plane around the center of a galaxy. Thus, $\dot{r}=0$ and $\dot{z}=0$. Let us further consider that the Hamiltonian is normalized to be equal to $-\frac{1}{2}$ for simplicity ${ }^{2}$ [28]. Hence, the system composed of the equations

$$
\begin{aligned}
\mathcal{H}+\frac{1}{2} & =0, \\
\frac{\partial}{\partial r}\left(\mathcal{H}+\frac{1}{2}\right) & =0,
\end{aligned}
$$

allows solutions to be obtained for the energy $E$ and the moment $L$ :

$$
\begin{aligned}
& E=E\left(r, \alpha, M, \alpha^{\prime}, M^{\prime}\right), \\
& L=L\left(r, \alpha, M, \alpha^{\prime}, M^{\prime}\right) .
\end{aligned}
$$

The solutions (42) and (43) applied in Eqs. (37) and (38) provide the angular velocity

\footnotetext{
${ }^{2}$ Notice that any non-vanishing normalization may be chosen.
}

$$
\begin{aligned}
\Omega_{ \pm} & =\frac{\mathrm{d} \varphi}{\mathrm{d} t}=\frac{\frac{\mathrm{d} \varphi}{\mathrm{d} \tau}}{\frac{\mathrm{d} t}{\mathrm{~d} \tau}}=\frac{\dot{\varphi}}{\dot{t}} \\
& =-\frac{2 \mathrm{e}^{2 \alpha} \alpha^{\prime}}{\mathrm{e}^{\alpha}\left(M^{\prime}+M \alpha^{\prime}\right) \pm \sqrt{-4 r \alpha^{\prime}\left(-1+r \alpha^{\prime}\right)+\mathrm{e}^{2 \alpha}\left(M^{\prime}-M \alpha^{\prime}\right)^{2}}} .
\end{aligned}
$$

With the metric (8) and considering the prescription established by Chandrasekhar $\mathrm{d} \tau^{2}=-\mathrm{d} s^{2}$ [29], we have

$$
\begin{aligned}
\mathrm{d} \tau^{2}= & \frac{\mathrm{e}^{2 \alpha} r^{2}}{-e^{2 \alpha} M^{2}+r^{2}} \mathrm{~d} t^{2}\left\{1-\frac{\mathrm{e}^{-4 \alpha+2 \beta}\left(-\mathrm{e}^{2 \alpha} M^{2}+r^{2}\right)}{r^{2}}\right. \\
& \times\left[\left(\frac{\mathrm{d} r}{\mathrm{~d} t}\right)^{2}+\left(\frac{\mathrm{d} z}{\mathrm{~d} t}\right)^{2}\right] \\
& \left.-\frac{\mathrm{e}^{-4 \alpha}\left(\mathrm{e}^{2 \alpha} M^{2}-r^{2}\right)^{2}\left(\frac{\mathrm{d} \varphi}{\mathrm{d} t}-\frac{\mathrm{e}^{3 \alpha} M}{-\mathrm{e}^{2 \alpha} M^{2}+r^{2}}\right)^{2}}{r^{2}}\right\} .
\end{aligned}
$$

Considering $u^{0}=\frac{\mathrm{d} t}{\mathrm{~d} \tau}$, Eq. (45) can be rewritten as

$$
1=\frac{\mathrm{e}^{2 \alpha} r^{2}}{-\mathrm{e}^{2 \alpha} M^{2}+r^{2}}\left(u^{0}\right)^{2}\left(1-v^{2}\right),
$$

where

$v^{2}=v_{r}^{2}+v_{z}^{2}+v_{\varphi}^{2}$

Therefore, the ratio between the tangential velocity $v_{\varphi}$ and the velocity of light $c$ can be written as

$\left(\frac{v_{\varphi}}{c}\right)_{\Omega_{ \pm} M_{ \pm}}=\sqrt{\frac{e^{-4 \alpha}\left(e^{2 \alpha} M_{ \pm}^{2}-r^{2}\right)^{2}\left(\Omega_{ \pm}-\frac{e^{3 \alpha} M_{ \pm}}{-e^{2 \alpha} M_{ \pm}^{2}+r^{2}}\right)^{2}}{r^{2}}}$.

Figure 2 shows the ratio $\frac{v_{\varphi}}{c}$ for different combinations between the functions $\Omega$ and $M$, as predicted by Eq. (48), 
Fig. 2 When $r_{\min }=1$,

$k_{1}=0.5, a_{C_{3}}=0.5, C_{4}=0.1$

and $C_{5}=-10$, different

combinations of the $\Omega$ and $M$

functions in Eq. (48) promote

distinct tangential velocity

functions. It is interesting to

note that all functions tend to

have the same constant velocity

$v_{\varphi_{\text {cst }}}$. A similar behavior can be

seen for when $\Omega=\Omega_{+}$and

$M=M_{+}$or $\Omega=\Omega_{-}$and

$M=M_{-}$in relation to the velocities of stars in galaxies of work [10], which tend to a constant value in the approximate range of three orders of magnitude

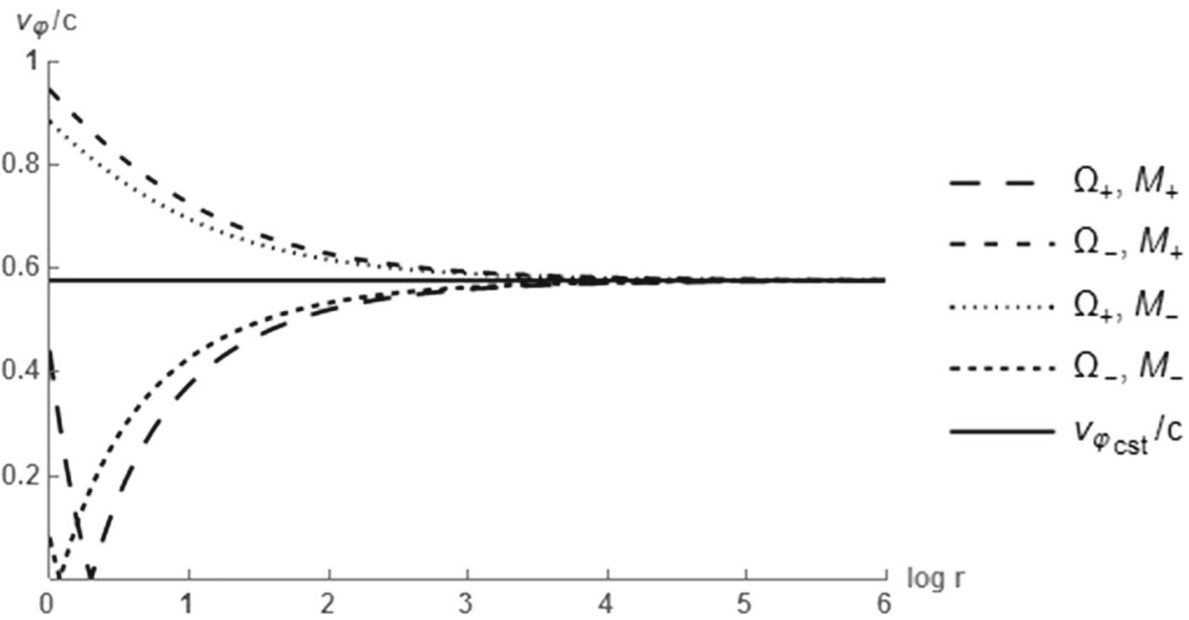

$R^{2}=1-\frac{\sum\left(v_{\varphi_{\mathrm{msr}}}-v_{\varphi}\right)^{2}}{\sum v_{\varphi_{\mathrm{msr}}}^{2}}$

when $r_{\min }=1, k_{1}=0.5, a_{C_{3}}=0.5, C_{4}=0.1$ and $C_{5}=$ -10 . It is important to notice that as $r$ increases the velocities tend to a single constant value

$\frac{v_{\varphi_{\mathrm{cst}}}}{c}=\sqrt{\frac{1-k_{1}}{1+k_{1}}}$,

which is determined when we compute the limit of the ratio $\frac{v \varphi}{c}$ for large $r$. This limit is represented in Fig. 2 by a solid line. The behavior of the tangential velocities for the cases where $\Omega=\Omega_{+}$and $M=M_{+}$or $\Omega=\Omega_{-}$and $M=M_{-}$is similar to that observed in relation to the velocities of the stars farthest from the centers of the 21 Sc type galaxies studied in Ref. [10]. In Ref. [10], the velocity growth is noticed as the distances from the stars to the centers of the galaxies grow by approximately 2-3 orders of magnitude, from 0.5 to $82.3 \mathrm{kpc}$, until they begin to tend to a constant value. This is similar to what is observed in Fig. 2, when the growth and consequent tendency for a constant velocity occurs in the range of about $10^{0}-10^{3} \mathrm{~m}$. This similarity of behaviors was the main motivation to determine if there would be a function (48) that would fit what was observed in Ref. [10].

For the necessary fit, we considered $C_{4}=1$ and the choice for the ranges of the constants $a_{C_{3}}, r_{\min }$ and the constant velocity $v_{\varphi_{\text {cst }}}$ from Eq. (49) was performed between those defined by Eqs. (50)-(52):

$$
\begin{aligned}
a_{C_{3}}= & 0.001 n, \quad n=1,2, \ldots, 999, \\
r_{\min }= & 10^{13.0+0.1 n}, \quad n=0,1, \ldots, 70, \\
v_{\varphi_{\mathrm{cst}}}= & \min v_{\varphi_{\mathrm{msr}}}+n, \quad n=0,1, \ldots, \\
& \max v_{\varphi_{\mathrm{msr}}}-\min v_{\varphi_{\mathrm{msr}}},
\end{aligned}
$$

where $v_{\varphi_{\mathrm{msr}}}$ are the measured velocities of the galaxy under study [10].

The coefficient of determination, $R^{2}$, is defined by Eq. (53): where $v_{\varphi}$ is the calculated velocity by Eq. (48), when $\Omega=$ $\Omega_{+}$and $M=M_{+}$. Although the coefficient of determination $R^{2}$ is not the most suitable for testing a non-linear regression model, it is adopted here as a standard definition for the values of the constants $a_{C_{3}}, r_{\min }$ and $v_{\varphi_{\mathrm{cst}}}$. The values chosen are those that result in the highest coefficient of determination with the uncorrected total sum of squares.

As for the constant $C_{5}$, it is related to $a_{C_{3}}, r_{\min }$ and $v_{\varphi_{\mathrm{cst}}}$, and it has been defined in such a way that CTC does not occur in the range of $0.5-82.3 \mathrm{kpc}$. For this to happen, the function $M=M_{+}$, Eq. (26), was rewritten as

$M(r)_{+}=C_{5} M_{1}(r)+M_{2}(r)$,

where

$M_{1}(r)=\sqrt{r^{1-k_{1}\left(C_{3}+r^{2 k_{1}}\right)},}$
$M_{2}(r)=\sqrt{-\frac{r^{1+3 k_{1}}}{C_{3} \mathrm{e}^{2 C_{4}}\left(C_{3}+r^{2 k_{1}}\right)}}$.

Thus,

$C_{5 g_{\varphi \varphi}>0}=-\frac{M_{2}\left(r=a_{C_{5}} r_{\min }\right)}{M_{1}\left(r=a_{C_{5}} r_{\min }\right)}$,

with $a_{C_{5}}=10^{6}$. When the best fit of the function (48) is sought for the 21 galaxies of Ref. [10], if the constant $C_{5}$ is calculated according to Eq. (57), the first term of Eq. (31) is always larger than the second term, and, therefore, there will be no CTC, because $g_{\varphi \varphi}>0$. However, the $C_{5}$ definition leads to $v_{\varphi} \geq v_{\varphi_{\text {cst }}}$ in this case, which runs counter to the observations, since we expect $v_{\varphi} \leq v_{\varphi_{\text {cst }}}$. For this problem to be solved, simply multiply $C_{5}$ by -1 , that is, use the same constant $C_{5}$ as determined before, but with a positive sign. 
The tangential velocity will have adequate values, but this time CTCs cannot be precluded, since $g_{\varphi \varphi}<0$. This happens because the values of $C_{5}$ for $v_{\varphi} \leq v_{\varphi_{\text {cst }}}$ are out of the range of $C_{5}$ where CTC does not appear. For example, the function (48) has its best fit to the measurements of the NGC 1087 galaxy when $\Omega=\Omega_{+}, M=M_{+}, C_{4}=1, a_{C 3}=0.723$, $r_{\min }=10^{16.0} \mathrm{~m}, v_{\varphi_{\mathrm{cst}}}=130 \mathrm{~km} / \mathrm{s}$ and

$$
\begin{aligned}
C_{5 v_{\varphi} \leq v_{\varphi_{\mathrm{cst}}}} & =+\frac{M_{2}\left(r=a_{C_{5}} r_{\min }\right)}{M_{1}\left(r=a_{C_{5}} r_{\min }\right)} \\
& =4.3266 \cdot 10^{-17} .
\end{aligned}
$$

With this fitting CTC occurs, because in order to have $g_{\varphi \varphi}>$ 0 it is necessary that

$-4.3267 \cdot 10^{-17}<C_{g_{\varphi \varphi}>0}<-4.3265 \cdot 10^{-17}$,

but this value leads to $v_{\varphi} \geq v_{\varphi_{\mathrm{cst}}}$.

To sum up, Eqs. (57) and (58) allow us to write the following relation:

$$
C_{5 g_{\varphi \varphi}>0}=-C_{5 v_{\varphi} \leq v_{\varphi_{\mathrm{cst}}}}
$$

If we obey Eq. (59), when

$$
\left|C_{5 g_{\varphi \varphi}>0}\right|=\left|C_{5 v_{\varphi} \leq v_{\varphi_{\mathrm{cst}}}}\right|,
$$

if $C_{5}<0$, there is no CTC, but $v_{\varphi} \geq v_{\varphi_{\text {cst }}}$; on the other hand, if $C_{5}>0, v_{\varphi} \leq v_{\varphi_{\mathrm{cst}}}$, but there is CTC.

Figure 3 shows the behavior of the fitted function (48) for the NGC 701, NGC 1087, NGC 3672 and NGC 801 galaxies, when $C_{5}>0$, that is, when $v_{\varphi}$ takes suitable values with the observations, but $g_{\varphi \varphi}<0$.

The confidence intervals (CIs) of the velocities were calculated according to Eq. (60):

$v_{\varphi_{\mathrm{CI}}}(r)=v_{\varphi}(r) \pm t\left(n-p, 1-\frac{1-\mathrm{CI}}{2}\right) s_{e}(r)$,

where $t$ is the $1-\frac{1-\mathrm{CI}}{2}$ quantile of the Student t-distribution with $n-p$ degrees of freedom, $n$ being the number of measurements taken for the galaxy and $p=1$, since all constants of the function (48) have been previously defined. Besides, the standard error $s_{e}$ is

$s_{e}(r)=\sqrt{s^{2}\left(1+\frac{v_{\varphi}(r)^{2}}{\sum v_{\varphi}^{2}}\right)}$,

with the estimated error variance $s^{2}$ equal to

$$
s^{2}=\frac{\sum\left(v_{\varphi_{\mathrm{msr}}}-v_{\varphi}\right)^{2}}{n-p} .
$$

CIs equal to $68 \%, 95 \%$ and $99 \%$ for the model applied in the four galaxies are represented in the Fig. 3. The values of the velocities $v_{\varphi_{\mathrm{msr}}}$ and $v_{\varphi}$, the standard errors $s_{e}$, and the CIs shown in the same figure are in Tables 3, 4, 5 and 6 of the appendix. In Table 1 we see the values of the constants with which the highest coefficient of determination $R^{2}$ was obtained, providing the best fit of the function (48) for each one of the four galaxies at hand. Among the 21 studied galaxies, NGC 701 and NGC 3672 are the ones with the lowest coefficients of determination, and NGC 1087 and NGC 801 are those with the highest coefficients. These two last galaxies have smaller standard errors than the two first, ensuring a better accuracy of the calculated velocities.

There is an alternative to the function (48) in order to suitably fit velocities with the observations and also not allow the existence of CTCs. But this implies the need of both $\mathrm{e}^{-C_{4}}$ and $C_{5}$ to assume pure imaginary values, which will make the solution (26) be pure imaginary number and the solutions (21) and (24) become complex, but the component $g_{\varphi \varphi}(31)$ and the tangential velocity (48) remain real, as well as the metric (8). So, if we now consider $\Omega=\Omega_{-}$instead of $\Omega=\Omega_{+}$, and, for example, $C_{4}=\frac{\pi}{2} \mathrm{i}$ and $C_{5}=\mathrm{i}, g_{\varphi \varphi}$ will be positive and the tangential velocity will assume values of the expected order of magnitude. The results for the best fits of the NGC 701, NGC 1087, NGC 3672 and NGC 801 galaxies for this last alternative can also be seen in Fig. 3 and in Table 1.

As for the metric (8), when $C_{4}=\frac{\pi}{2} \mathrm{i}$, the component $g_{t t}$ reads

$g_{t t}=r^{1-k_{1}}\left(C_{3}+r^{2 k_{1}}\right)$

For the fit of the function (48), the conditions (27) and (28) were maintained for the metric to be real. Thus, $C_{3}+r^{2 k_{1}}>$ 0 , which leads to $g_{t t}>0$. Having still $g_{\varphi \varphi}>0$ and the other spatial components $g_{r r}$ and $g_{z z}$ also positive, the metric (8) becomes an Euclidean metric.

\section{The ratio $\frac{\beta}{\alpha}$ : An alternative to explain the rotational curves?}

As we make use of a nearly cylindrical symmetry, it is necessary that the ratio between the functions (21) and (24) gives $\frac{\beta}{\alpha}=C_{\alpha \beta} \approx 2$. Since we are interested in the behavior of $v_{\varphi}$ for the $r=0.5-82.3 \mathrm{kpc}$ range, it is important to calculate the limit for large $r$ of the $\frac{\beta}{\alpha}$ ratio. This limit is constant and can be written as

$C_{\alpha \beta_{\mathrm{cst}}}=1-\frac{2 C_{a}}{1+k_{1}}$, 
Fig. 3 Stars velocities of the NGC 701, NGC 1087, NGC 3672 and NGC 801 galaxies [10] compared to the best fit of the function (48). When $\Omega=\Omega_{+}$, the curves show the fits for when the $C_{4}$ and $C_{5}$ constants are real and there is CTC in the range represented in the graph, because $g_{\varphi \varphi}<0$; when $\Omega=\Omega_{-}, C_{4}$ and $C_{5}$ are imaginary, $g_{\varphi \varphi}>0$, and there is no CTC, and the metric is Euclidean, because $g_{t t}>0$. The values of the constants and the coefficient of determination for each galaxy are in the Table 1, and the values of the measured and calculated velocities, the standard errors and the CIs are in Tables 3-6 of the appendix

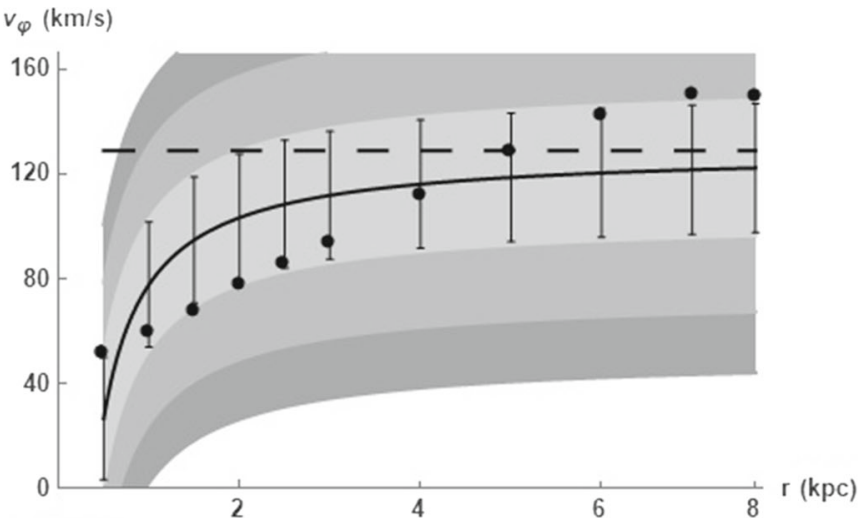

$v_{\varphi}(\mathrm{km} / \mathrm{s})$

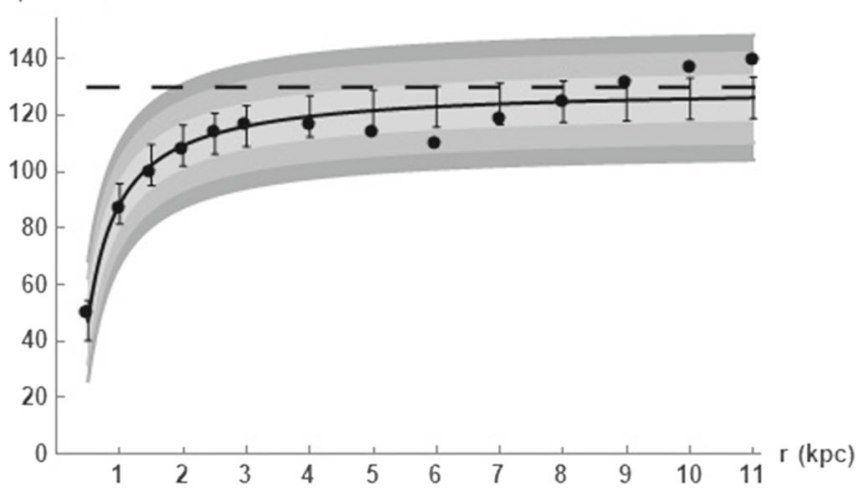

- $v_{\varphi_{\mathrm{msr}}}(\mathrm{NGC} 1087)$

$$
\begin{aligned}
& -v_{\varphi}\left(\Omega_{ \pm}, M_{+}\right) \\
& --v_{\varphi_{\text {cst }}}\left(\Omega_{ \pm}, M_{+}\right) \\
& I s_{e} \\
& \square \mathrm{Cl}=68 \% \\
& \square \mathrm{Cl}=95 \% \\
& \square \mathrm{Cl}=99 \%
\end{aligned}
$$

$v_{\varphi}(\mathrm{km} / \mathrm{s})$

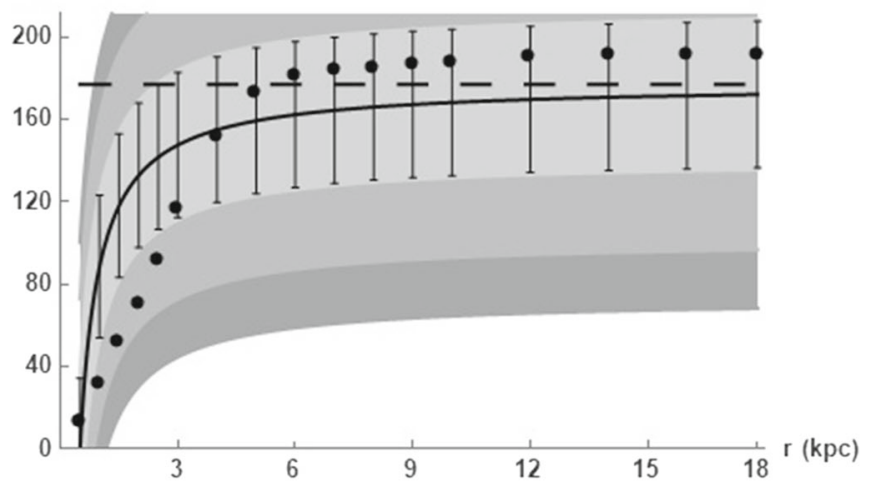

- $v_{\varphi_{\mathrm{msr}}}(\mathrm{NGC} 3672)$

$-v_{\varphi}\left(\Omega_{ \pm}, M_{+}\right)$

$-v_{\varphi_{\text {cst }}}\left(\Omega_{ \pm}, M_{+}\right)$

$I s_{e}$

( $\mathrm{Cl}=68 \%$

$\mathrm{Cl}=95 \%$

- $\mathrm{Cl}=99 \%$

$v_{\varphi}(\mathrm{km} / \mathrm{s})$

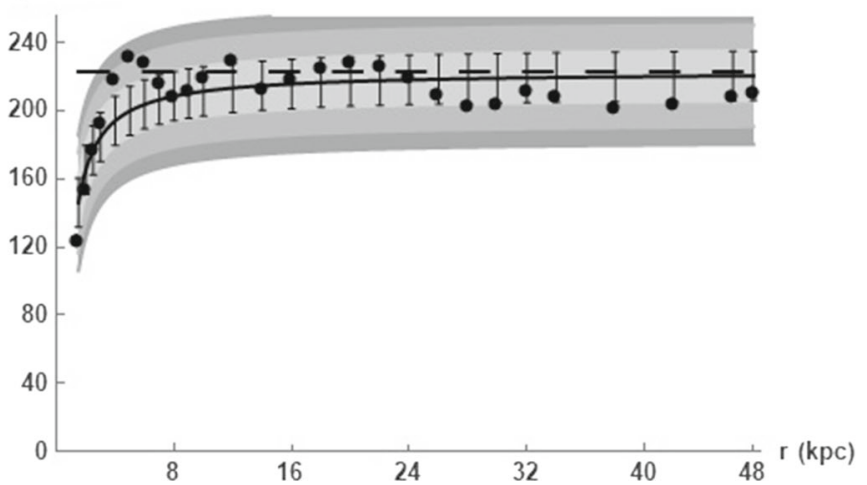

- $v_{\varphi_{\mathrm{msr}}}$ (NGC 801)

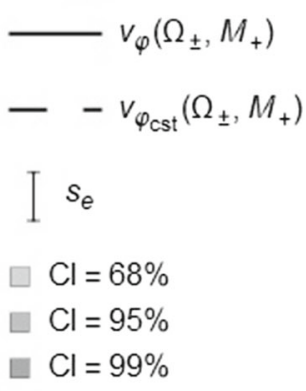


Table 1 Values of the

components $g_{t t}$ and $g_{\varphi \varphi}$, the constants and the determination coefficients for the fits of the function (48) made for NGC 701, NGC 1087, NGC 3672 and NGC 801 galaxies, shown in

Fig. 3. $R_{\text {farthest }}$ is the radial distance of outermost measured velocity. When the constant $C_{5}$ is real, it is defined by Eq. (58)

\begin{tabular}{lllllllll}
\hline & NGC 701 & \multicolumn{3}{l}{ NGC 1087 } & \multicolumn{2}{l}{ NGC 3672 } & \multicolumn{3}{l}{ NGC 801 } \\
\hline$R_{\text {farthest }}(\mathrm{kpc})$ & 7.7 & 7.7 & 11.0 & 11.0 & 17.8 & 17.8 & 47.4 & 47.4 \\
$\Omega$ & $\Omega_{+}$ & $\Omega_{-}$ & $\Omega_{+}$ & $\Omega_{-}$ & $\Omega_{+}$ & $\Omega_{-}$ & $\Omega_{+}$ & $\Omega_{-}$ \\
$M$ & $M_{+}$ & $M_{+}$ & $M_{+}$ & $M_{+}$ & $M_{+}$ & $M_{+}$ & $M_{+}$ & $M_{+}$ \\
$v_{\varphi_{\text {cst }}}(\mathrm{km} / \mathrm{s})$ & 129 & 129 & 130 & 130 & 177 & 177 & 223 & 223 \\
$\log r_{\text {min }}$ & 16.1 & 15.8 & 16.0 & 15.8 & 16.5 & 16.1 & 16.5 & 16.3 \\
$a_{C_{3}}$ & 0.699 & 0.696 & 0.723 & 0.454 & 0.331 & 0.522 & 0.562 & 0.353 \\
$C_{4}$ & 1 & $\frac{\pi}{2} \mathrm{i}$ & 1 & $\frac{\pi}{2} \mathrm{i}$ & 1 & $\frac{\pi}{2} \mathrm{i}$ & 1 & $\frac{\pi}{2} \mathrm{i}$ \\
$C_{5}$ & $\frac{M_{2}}{M_{1}}$ & $\mathrm{i}$ & $\frac{M_{2}}{M_{1}}$ & $\mathrm{i}$ & $\frac{M_{2}}{M_{1}}$ & $\mathrm{i}$ & $\frac{M_{2}}{M_{1}}$ & $\mathrm{i}$ \\
$R^{2}$ & 0.958 & 0.958 & 0.996 & 0.996 & 0.953 & 0.953 & 0.996 & 0.996 \\
$g_{\varphi \varphi}$ & $<0$ & $>0$ & $<0$ & $>0$ & $<0$ & $>0$ & $<0$ & $>0$ \\
$g_{t t}$ & $<0$ & $>0$ & $<0$ & $>0$ & $<0$ & $>0$ & $<0$ & $>0$ \\
\hline
\end{tabular}

leading to

$C_{a}=-\frac{1}{2}\left(C_{\alpha \beta_{\mathrm{cst}}}-1\right)\left(1+k_{1}\right)$.

According to Eq. (65), when $C_{\alpha \beta_{\mathrm{cst}}}=2.002$ (that is, $1 \%$ bigger than 2) and, for example, $v_{\varphi_{\mathrm{cst}}}=200 \mathrm{~km} / \mathrm{s}$ (this
$C_{a}=-\frac{1}{2}(2.002-1)\left(1+k_{1}\right)$.

By Eq. (25), $k_{1} \geq 0$. So, the only valid solution for the system with Eqs. (66) and (67) is $k_{1}=3.004$. This last result leads to $v_{\varphi_{\mathrm{cst}}}=0.707 \mathrm{ci}$, which is not possible, because $v_{\varphi_{\mathrm{cst}}}$ must be real.

With the line element in the Jordan-Fierz frame (3), it is also possible to determine the angular velocity ${ }^{3}$ :

$\Omega_{ \pm}= \pm \frac{\mathrm{e}^{5 \alpha} \Xi\left(2 \Lambda \alpha^{\prime}+\Lambda^{\prime}\right)}{\sqrt{\mathrm{e}^{8 \alpha} \Lambda^{2} \Xi^{4}-\mathrm{e}^{6 \alpha} r \Xi^{2}\left(2 \Lambda \alpha^{\prime}+\Lambda^{\prime}\right)\left[2 \Lambda\left(-1+r \alpha^{\prime}\right)-r \Lambda^{\prime}\right]} \mp \mathrm{e}^{4 \alpha} \Xi\left(M \alpha^{\prime}-\Lambda \Xi\right)}$,

value has the suitable order of magnitude, because, for all 21 studied galaxies, $96 \mathrm{~km} / \mathrm{s} \leq v_{\varphi_{\text {cst }}} \leq 266 \mathrm{~km} / \mathrm{s}$ ), with $c=3 \cdot 10^{5} \mathrm{~km} / \mathrm{s}$ and

$\frac{v_{\varphi_{\mathrm{cst}}}}{c}=\sqrt{\frac{1-k_{1}}{1+k_{1}}}$

the constant $C_{a}$ equals -1.002 . Thus, considering

$k_{1}=\sqrt{1-4 C_{1}^{2}-4 C_{a}} \geq 0$

we have $C_{1}= \pm 1.001 \neq 0$, that is, the scalar field $\phi$ is not constant (see Eq. (16)), which indicates that the behavior of tangential velocities of the stars seen so far cannot be described by GR, and the use of BD gravitation is indeed required.

If the modeling option is in the framework of the GR gravity, i.e., with $C_{1}=0$, by Eqs. (25) and (65), with $C_{\alpha \beta_{\text {cst }}}=2.002$, we have

$C_{a}=\frac{1-k_{1}^{2}}{4}$, where

$$
\begin{aligned}
& \Lambda=\mathrm{e}^{2 \kappa \phi}, \\
& \Xi=M \alpha^{\prime}-M^{\prime} .
\end{aligned}
$$

When we insert the velocity (68) in the Eq. (48), we have the ratio $\frac{v_{\varphi}}{c}$ in the Jordan-Fierz frame, where the parameter $\omega$ now appears explicitly. In this case, the limit of this ratio for large $r$ tends to the single value

$$
\frac{v_{\varphi_{\mathrm{cst}}}}{c}=\sqrt{\frac{\left(1-k_{1}^{2}\right)(3+2 \omega)+4 C_{1}\left(C_{1}+\sqrt{3+2 \omega}\right)}{\left(1+k_{1}\right)^{2}(3+2 \omega)+4 C_{1}\left[C_{1}+\left(1+k_{1}\right) \sqrt{3+2 \omega}\right]}} .
$$

When $\omega \rightarrow \infty$, the limit of this ratio is

$\lim _{\omega \rightarrow \infty} \frac{v_{\varphi_{\mathrm{cst}}}}{c}=\sqrt{\frac{1-k_{1}}{1+k_{1}}}$

This last result is identical to the one found for the ratio $\frac{v_{\varphi_{\text {cst }}}}{c}$ in the Einstein frame (see Eq. (49)). This happens because

\footnotetext{
3 Along the calculations we have considered $\kappa$ as well $\omega$ as positive parameters.
} 


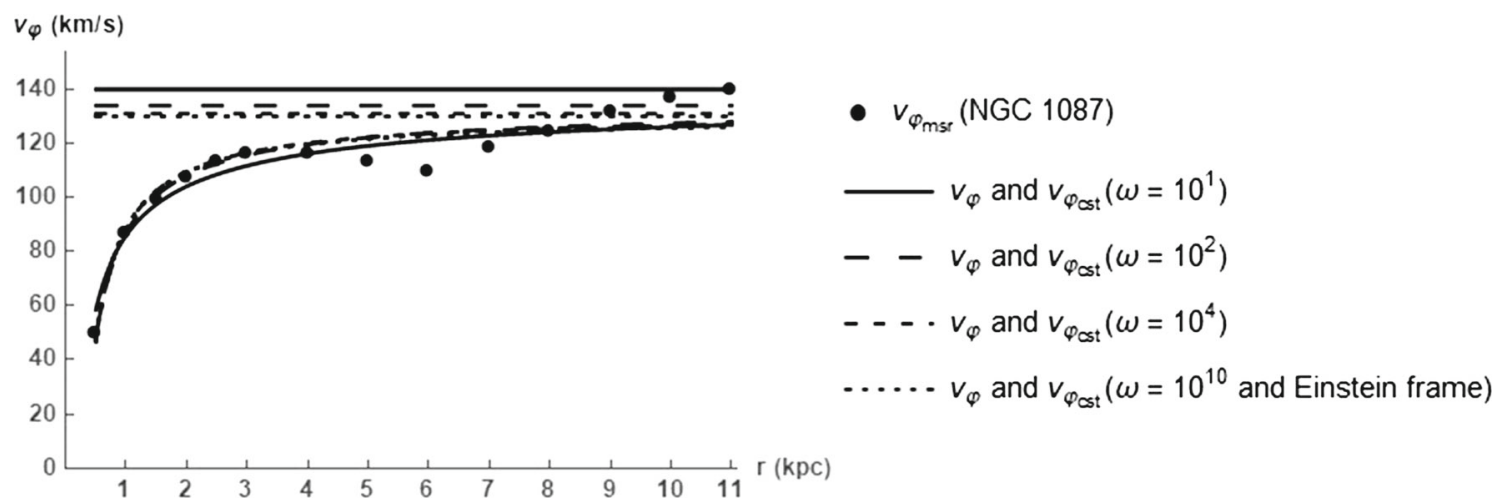

Fig. 4 Stars velocities of the NGC 1087 galaxy [10] compared to the best fits of the function (48) in the Jordan-Fierz frame. The values of the constants and the coefficients of determination are in the Table 2. From $\omega=10^{2}$, the fit of the function (48) results in almost identical curves

Table 2 Values of the constants and the coefficients of determination for the fits of the function (48) in the Jordan-Fierz for NGC 1087 galaxy, shown in Fig. 4. The constant $C_{1}$ was defined by the system of Eqs. (25), (65) and (71), and $C_{5}$ was defined by Eq. (58)

\begin{tabular}{|c|c|c|c|c|c|c|c|c|c|c|c|c|}
\hline \multicolumn{13}{|c|}{ NGC 1087} \\
\hline $\log \omega$ & $\Omega$ & $M$ & $v_{\varphi_{\mathrm{cst}}}(\mathrm{km} / \mathrm{s})$ & $\log r_{\text {min }}$ & $a_{C_{3}}$ & $C_{1}$ & $C_{2}$ & $C_{4}$ & $C_{5}$ & $C_{\alpha \beta_{\mathrm{cst}}}$ & $R^{2}$ & $g_{\varphi \varphi}$ \\
\hline 1 & $\Omega_{+}$ & $M_{+}$ & 140 & 13.8 & 0.697 & $f\left(\omega, v_{\varphi_{\mathrm{cst}}}, C_{\alpha \beta_{\mathrm{cst}}}\right)$ & 1 & 1 & $\frac{M_{2}}{M_{1}}$ & 2.002 & 0.996 & $<0$ \\
\hline 2 & $\Omega_{+}$ & $M_{+}$ & 134 & 15.7 & 0.324 & $f\left(\omega, v_{\varphi_{\mathrm{cst}}}, C_{\alpha \beta_{\mathrm{cst}}}\right)$ & 1 & 1 & $\frac{M_{2}}{M_{1}}$ & 2.002 & 0.997 & $<0$ \\
\hline 4 & $\Omega_{+}$ & $M_{+}$ & 131 & 16.1 & 0.387 & $f\left(\omega, v_{\varphi_{\mathrm{cst}}}, C_{\alpha \beta_{\mathrm{cst}}}\right)$ & 1 & 1 & $\frac{M_{2}}{M_{1}}$ & 2.002 & 0.997 & $<0$ \\
\hline 10 & $\Omega_{+}$ & $M_{+}$ & 130 & 16.0 & 0.723 & $f\left(\omega, v_{\varphi_{\mathrm{cst}}}, C_{\alpha \beta_{\mathrm{cst}}}\right)$ & 1 & 1 & $\frac{M_{2}}{M_{1}}$ & 2.002 & 0.996 & $<0$ \\
\hline
\end{tabular}

when $\omega \rightarrow \infty, \tilde{g}_{\mu \nu}=g_{\mu \nu}$. Thus, for a large $\omega$, the behaviors of the velocity $v_{\varphi}$ are the same in the Einstein and JordanFierz frames. For example, the Fig. 4 shows the behavior of the fitted velocity $v_{\varphi}$ for the NGC 1087 galaxy, with different values of $\omega$. We consider $C_{2}=C_{4}=1 ; C_{\alpha \beta_{\text {cst }}}=2.002$; $a_{C 3}, r_{\min }$ and $v_{\varphi_{\mathrm{cst}}}$ defined by Eqs. (50)-(52); $C_{5}$ defined by Eq. (58); $C_{1}$ determined by the system formed by Eqs. (25), (65) and (71), depending on the constants $\omega, v_{\varphi_{\text {cst }}}$ and $C_{\alpha \beta}$.

As $\omega$ grows, the behavior of the velocity $v_{\varphi}$ is close to the one found in the Einstein frame (also shown in Fig. 3). For instance, when $\omega=10^{10}$, the constants $a_{C 3}, r_{\min }$ and $v_{\varphi_{\mathrm{cst}}}$ coincide in both frames (see Tables 1 and 2). It is also interesting to note that, for $\omega=10^{2}$ or $\omega=10^{4}$, the coefficient of determination in the Jordan-Fierz is somewhat higher than that found when $\omega=10^{10}$ or in the Einstein frame.

As usual, when using the Jordan-Fierz frame along with a large $\omega$, the BD gravitation must provide (almost) the same results of GR. Nevertheless, the obtained good results with a small $\omega \simeq 10^{2}$ attest that such gravitation is the correct option, reinforcing what was already argued in the first part of this section. In addition, because of the difference between the fitted curves in the different frames, accurate measurements of the stars velocities may indicate which of the two frames would be most appropriate for the quantitative description of the rotational curves of the galaxies.

\section{Final remarks}

A complete spacetime analysis was performed taking into account a nearly cylindrically symmetry in the context of the BD gravitation. The resulting spacetime is regular and there is a possibility of the existing of CTCs for a certain range of the coordinate $r$. With an appropriate choice of the integration constants, it is possible to avoid the CTCs.

The solution presented in this paper is shown to be suitable to generate the rotational curves of the galaxies, because it reproduces the velocity growth behavior in the order of magnitude compatible with the experimental data [10]. For this purpose, the solution indicates that it is necessary to admit the existence of CTCs in a certain range. We notice, however, that this type of possibility is far from consensual. On the one hand the chronological protection conjecture says that the laws of physics prohibit the emergence of CTCs [30]. On the other hand, in interesting work it was pointed out that the same laws allow for the CTCs and they can appear naturally [31-35].

Returning to the main goal of our paper, we present a model in which CTCs are ruled out, but the metric must be Euclidean. Theories of quantum gravity, which seek to describe phenomena of the time of grand unification theory (GUT), have indeed made use of such a stratagem [36,37]. 
Finally, we would like to stress that the BD gravity adequately describes the observational data as an effective theory of gravity coming from an yet unknown complete theory of a quantum gravity.

Acknowledgements JMHS would like to thank the CNPq for partial support (Grant numbers 304629/2015-4; 445385/2014-6). The authors would like to thank R. Floriano da Silva for his collaboration in calculating the solutions for the metric's functions and O. Grichtchouk for a critical reading of this manuscript.

Open Access This article is distributed under the terms of the Creative Commons Attribution 4.0 International License (http://creativecomm ons.org/licenses/by/4.0/), which permits unrestricted use, distribution, and reproduction in any medium, provided you give appropriate credit to the original author(s) and the source, provide a link to the Creative
Commons license, and indicate if changes were made. Funded by SCOAP ${ }^{3}$.

Appendix: Measured and calculated velocities, standard errors and confidence intervals for the NGC 701, NGC 1087, NGC 3672 and NGC 801 galaxies

See Tables 3, 4, 5 and 6 .
Table 3 Measured and calculated velocities, standard errors and confidence intervals for the NGC 701 galaxy

Table 4 Measured and calculated velocities, standard errors and confidence intervals for the NGC 1087 galaxy

\begin{tabular}{|c|c|c|c|c|c|c|}
\hline \multicolumn{7}{|l|}{ NGC 701} \\
\hline \multirow[t]{2}{*}{$r(\mathrm{kpc})[10]$} & \multirow[t]{2}{*}{$v_{\varphi_{\mathrm{msr}}}(\mathrm{km} / \mathrm{s})[10]$} & \multirow[t]{2}{*}{$v_{\varphi}(\mathrm{km} / \mathrm{s})$} & \multirow[t]{2}{*}{$s_{e}(\mathrm{~km} / \mathrm{s})$} & \multicolumn{3}{|c|}{ Confidence interval } \\
\hline & & & & $68 \%$ & $95 \%$ & $99 \%$ \\
\hline 0.5 & 52 & 27 & 23 & {$[2,51]$} & {$[-25,79]$} & {$[-47,101]$} \\
\hline 1 & 60 & 78 & 24 & {$[53,103]$} & {$[25,131]$} & {$[2,153]$} \\
\hline 1.5 & 68 & 95 & 24 & {$[70,120]$} & {$[41,149]$} & {$[18,171]$} \\
\hline 2 & 78 & 103 & 24 & {$[78,129]$} & {$[49,157]$} & {$[27,180]$} \\
\hline 2.5 & 86 & 109 & 24 & {$[83,134]$} & {$[54,163]$} & {$[31,186]$} \\
\hline 3 & 94 & 112 & 24 & {$[86,137]$} & {$[58,166]$} & {$[35,189]$} \\
\hline 4 & 112 & 116 & 25 & {$[91,142]$} & {$[62,171]$} & {$[39,194]$} \\
\hline 5 & 129 & 119 & 25 & {$[93,144]$} & {$[64,173]$} & {$[41,197]$} \\
\hline 6 & 143 & 120 & 25 & {$[95,146]$} & {$[66,175]$} & {$[43,198]$} \\
\hline 7 & 151 & 122 & 25 & {$[96,147]$} & {$[67,177]$} & {$[44,200]$} \\
\hline 7.7 & 150 & 122 & 25 & {$[97,148]$} & {$[67,177]$} & {$[44,200]$} \\
\hline
\end{tabular}

\begin{tabular}{|c|c|c|c|c|c|c|}
\hline \multicolumn{7}{|l|}{ NGC 1087} \\
\hline \multirow[t]{2}{*}{$r(\mathrm{kpc})[10]$} & \multirow[t]{2}{*}{$v_{\varphi_{\mathrm{msr}}}(\mathrm{km} / \mathrm{s})[10]$} & \multirow[t]{2}{*}{$v_{\varphi}(\mathrm{km} / \mathrm{s})$} & \multirow[t]{2}{*}{$s_{e}(\mathrm{~km} / \mathrm{s})$} & \multicolumn{3}{|c|}{ Confidence interval } \\
\hline & & & & $68 \%$ & $95 \%$ & $99 \%$ \\
\hline 0.5 & 50 & 47 & 7 & {$[40,55]$} & {$[32,63]$} & {$[26,69]$} \\
\hline 1 & 87 & 89 & 7 & {$[81,96]$} & {$[73,104]$} & {$[67,110]$} \\
\hline 1.5 & 100 & 102 & 7 & {$[95,110]$} & {$[87,118]$} & {$[81,124]$} \\
\hline 2 & 108 & 109 & 7 & {$[102,117]$} & {$[94,125]$} & {$[88,131]$} \\
\hline 2.5 & 114 & 113 & 7 & {$[106,121]$} & {$[98,129]$} & {$[92,135]$} \\
\hline 3 & 117 & 116 & 7 & {$[109,124]$} & {$[101,132]$} & {$[94,138]$} \\
\hline 4 & 117 & 120 & 7 & {$[112,127]$} & {$[104,135]$} & {$[98,142]$} \\
\hline 5 & 114 & 122 & 7 & {$[114,129]$} & {$[106,137]$} & {$[100,144]$} \\
\hline 6 & 110 & 123 & 7 & {$[116,131]$} & {$[107,139]$} & {$[101,145]$} \\
\hline 7 & 119 & 124 & 7 & {$[117,132]$} & {$[108,140]$} & {$[102,146]$} \\
\hline 8 & 125 & 125 & 7 & {$[117,132]$} & {$[109,141]$} & {$[103,147]$} \\
\hline 9 & 132 & 125 & 7 & {$[118,133]$} & {$[110,141]$} & {$[103,147]$} \\
\hline 10 & 137 & 126 & 7 & {$[118,133]$} & {$[110,142]$} & {$[104,148]$} \\
\hline 11 & 140 & 126 & 7 & {$[119,134]$} & {$[110,142]$} & {$[104,148]$} \\
\hline
\end{tabular}


Table 5 Measured and calculated velocities, standard errors and confidence intervals for the NGC 3672 galaxy

Table 6 Measured and calculated velocities, standard errors and confidence intervals for the NGC 801 galaxy

\begin{tabular}{|c|c|c|c|c|c|c|}
\hline \multicolumn{7}{|l|}{ NGC 3672} \\
\hline \multirow[t]{2}{*}{$r(\mathrm{kpc})[10]$} & \multirow[t]{2}{*}{$v_{\varphi_{\mathrm{msr}}}(\mathrm{km} / \mathrm{s})[10]$} & \multirow[t]{2}{*}{$v_{\varphi}(\mathrm{km} / \mathrm{s})$} & \multirow[t]{2}{*}{$s_{e}(\mathrm{~km} / \mathrm{s})$} & \multicolumn{3}{|c|}{ Confidence interval } \\
\hline & & & & $68 \%$ & $95 \%$ & $99 \%$ \\
\hline 0.5 & 14 & 0.02 & 34 & {$[-35,35]$} & {$[-73,73]$} & {$[-100,100]$} \\
\hline 1 & 32 & 89 & 35 & {$[53,124]$} & {$[15,162]$} & {$[-13,190]$} \\
\hline 1.5 & 52 & 118 & 35 & {$[82,154]$} & {$[44,192]$} & {$[16,220]$} \\
\hline 2 & 71 & 133 & 35 & {$[97,169]$} & {$[58,207]$} & {$[30,235]$} \\
\hline 2.5 & 92 & 142 & 35 & {$[106,178]$} & {$[67,216]$} & {$[39,244]$} \\
\hline 3 & 117 & 148 & 35 & {$[111,184]$} & {$[73,222]$} & {$[45,250]$} \\
\hline 4 & 152 & 155 & 35 & {$[119,191]$} & {$[80,230]$} & {$[52,258]$} \\
\hline 5 & 173 & 159 & 35 & {$[123,196]$} & {$[84,234]$} & {$[56,263]$} \\
\hline 6 & 182 & 162 & 35 & {$[126,199]$} & {$[87,237]$} & {$[59,266]$} \\
\hline 7 & 184 & 164 & 35 & {$[128,201]$} & {$[89,240]$} & {$[61,268]$} \\
\hline 8 & 185 & 166 & 35 & {$[130,202]$} & {$[91,241]$} & {$[62,270]$} \\
\hline 9 & 187 & 167 & 36 & {$[131,204]$} & {$[92,242]$} & {$[63,271]$} \\
\hline 10 & 188 & 168 & 36 & {$[132,205]$} & {$[93,243]$} & {$[64,272]$} \\
\hline 12 & 191 & 170 & 36 & {$[133,206]$} & {$[94,245]$} & {$[66,273]$} \\
\hline 14 & 192 & 171 & 36 & {$[134,207]$} & {$[95,246]$} & {$[67,275]$} \\
\hline 16 & 192 & 171 & 36 & {$[135,208]$} & {$[96,247]$} & {$[68,275]$} \\
\hline 17.8 & 192 & 172 & 36 & {$[136,209]$} & {$[97,247]$} & {$[68,276]$} \\
\hline
\end{tabular}

\begin{tabular}{|c|c|c|c|c|c|c|}
\hline \multicolumn{7}{|l|}{ NGC 801} \\
\hline \multirow[t]{2}{*}{$r(\mathrm{kpc})[10]$} & \multirow[t]{2}{*}{$v_{\varphi_{\mathrm{msr}}}(\mathrm{km} / \mathrm{s})[10]$} & \multirow[t]{2}{*}{$v_{\varphi}(\mathrm{km} / \mathrm{s})$} & \multirow[t]{2}{*}{$s_{e}(\mathrm{~km} / \mathrm{s})$} & \multicolumn{3}{|c|}{ Confidence interval } \\
\hline & & & & $68 \%$ & $95 \%$ & $99 \%$ \\
\hline 1.5 & 123 & 146 & 14 & {$[132,161]$} & {$[117,176]$} & {$[106,186]$} \\
\hline 2 & 154 & 165 & 14 & {$[151,180]$} & {$[136,195]$} & {$[126,205]$} \\
\hline 2.5 & 177 & 177 & 14 & {$[162,191]$} & {$[147,206]$} & {$[137,217]$} \\
\hline 3 & 193 & 185 & 14 & {$[170,199]$} & {$[155,214]$} & {$[145,225]$} \\
\hline 4 & 218 & 194 & 14 & {$[180,209]$} & {$[165,224]$} & {$[154,234]$} \\
\hline 5 & 232 & 200 & 14 & {$[185,215]$} & {$[170,230]$} & {$[160,240]$} \\
\hline 6 & 228 & 204 & 14 & {$[189,218]$} & {$[174,233]$} & {$[164,244]$} \\
\hline 7 & 216 & 207 & 14 & {$[192,221]$} & {$[177,236]$} & {$[166,247]$} \\
\hline 8 & 208 & 209 & 14 & {$[194,223]$} & {$[179,238]$} & {$[168,249]$} \\
\hline 9 & 212 & 210 & 14 & {$[196,225]$} & {$[181,240]$} & {$[170,250]$} \\
\hline 10 & 220 & 211 & 14 & {$[197,226]$} & {$[182,241]$} & {$[171,252]$} \\
\hline 12 & 230 & 213 & 14 & {$[199,228]$} & {$[184,243]$} & {$[173,254]$} \\
\hline 14 & 213 & 215 & 14 & {$[200,229]$} & {$[185,244]$} & {$[175,255]$} \\
\hline 16 & 218 & 216 & 14 & {$[201,230]$} & {$[186,245]$} & {$[176,256]$} \\
\hline 18 & 225 & 217 & 14 & {$[202,231]$} & {$[187,246]$} & {$[176,257]$} \\
\hline 20 & 228 & 217 & 14 & {$[203,232]$} & {$[188,247]$} & {$[177,257]$} \\
\hline 22 & 226 & 218 & 14 & {$[203,232]$} & {$[188,247]$} & {$[178,258]$} \\
\hline 24 & 220 & 218 & 14 & {$[204,233]$} & {$[188,248]$} & {$[178,258]$} \\
\hline 26 & 209 & 219 & 14 & {$[204,233]$} & {$[189,248]$} & {$[178,259]$} \\
\hline 28 & 203 & 219 & 14 & {$[204,234]$} & {$[189,249]$} & {$[179,259]$} \\
\hline
\end{tabular}


Table 6 continued

\begin{tabular}{|c|c|c|c|c|c|c|}
\hline \multicolumn{7}{|l|}{ NGC 801} \\
\hline \multirow[t]{2}{*}{$r(\mathrm{kpc})[10]$} & \multirow{2}{*}{$v_{\varphi_{\mathrm{msr}}}(\mathrm{km} / \mathrm{s})[10]$} & \multirow[t]{2}{*}{$v_{\varphi}(\mathrm{km} / \mathrm{s})$} & \multirow[t]{2}{*}{$s_{e}(\mathrm{~km} / \mathrm{s})$} & \multicolumn{3}{|c|}{ Confidence interval } \\
\hline & & & & $68 \%$ & $95 \%$ & $99 \%$ \\
\hline 30 & 204 & 219 & 14 & {$[204,234]$} & {$[189,249]$} & {$[179,259]$} \\
\hline 32 & 212 & 219 & 14 & {$[205,234]$} & {$[190,249]$} & {$[179,260]$} \\
\hline 34 & 208 & 220 & 14 & {$[205,234]$} & {$[190,249]$} & {$[179,260]$} \\
\hline 38 & 202 & 220 & 14 & {$[205,235]$} & {$[190,250]$} & {$[180,260]$} \\
\hline 42 & 204 & 220 & 14 & {$[206,235]$} & {$[191,250]$} & {$[180,260]$} \\
\hline 46 & 208 & 220 & 14 & {$[206,235]$} & {$[191,250]$} & {$[180,261]$} \\
\hline 47.4 & 211 & 221 & 14 & {$[206,235]$} & {$[191,250]$} & {$[180,261]$} \\
\hline
\end{tabular}

\section{References}

1. C. Brans, R.H. Dicke, Phys. Rev. 124, 925 (1961)

2. B. Bertotti, L. Iess, P. Tortora, Nature 425, 374 (2003)

3. M.W. Clifford, Living Rev. Relat. 9, 3 (2006)

4. Th Damour, K. Nordtvedt, Phys. Rev. Lett. 70, 2217 (1993)

5. Th Damour, K. Nordtvedt, Phys. Rev. D 48, 3436 (1993)

6. Th Damour, A.M. Polyakov, Nucl. Phys. B 423, 532 (1994)

7. C. Contaldi, M.B. Hindmarsh, J. Magueijo, Phys. Rev. Lett. 82, 2034 (1999)

8. F. Zwicky, Helvetica Phys. Acta 6, 110 (1933)

9. F. Zwicky, Astrophys. J. 86, 217 (1937)

10. V.C. Rubin, W.K. Ford Jr., N. Thonnard, Astrophys. J. 238, 471 (1980)

11. M. Persic, P. Salucci, Astrophys. J. Suppl. Ser. 99, 501 (1995)

12. P. Salucci, M. Persic, Proc. of the Sesto DM1996 Conference (1997)

13. T. Matos, F.S. Guzmán, L.A. Ureña-López, D. Núñez, Scalar field dark matter, in Exact Solutions and Scalar Fields in Gravity-Recent Developments, ed. by A. Macias, J.L. Cervantes-Cota, C. Lämmerzahl (Kluwer Academic Publishers, Dordrecht, 2002)

14. T.H. Lee, B.J. Lee, Phys. Rev. D 69, 127502 (2004)

15. M. Leineker Costa, A.L. Naves de Oliveira, M.E.X. Guimarães, Int. J. Mod. Phys. D 15, 387 (2006)

16. B. Jensen, Class. Quantum Grav. 9, L7 (1992)

17. S. Mittmann Santos, J. M. Hoff Silva, J. L. Cindra, Straight spinning cosmic strings in Brans-Dicke gravity. arXiv: 1708.00507

18. S. Deser, R. Jackiw, Comments Nucl. Part. Phys. 20, 337 (1992)

19. P.O. Mazur, Phys. Rev. Lett. 57, 929 (1986)

20. V.A. Kostelecký, S. Samuel, Phys. Rev. D 39, 683 (1989)
21. V.A. Kostelecký, R. Potting, Nucl. Phys. B 359, 545 (1991)

22. V.A. Kostelecký, R. Potting, Phys. Rev. D 51, 3923 (1995)

23. V.A. Kostelecký, R. Potting, Phys. Lett. B 381, 89 (1996)

24. V. Faraoni, E. Gunzig, P. Nardone, Fund. Cosmic Phys. 20, 121 (1999)

25. I. Quiros, R. Garcia-Salcedo, J.E.M. Aguilar, T. Matos, Gen. Rel. Grav. 45, 489 (2013)

26. B. Boisseau, B. Linet, Gen. Rel. Grav. 30, 963 (1998)

27. K. Gödel, Rev. Mod. Phys. 21, 447 (1949)

28. T. Matos, D. Núñez, F.S. Guzmán, E. Ramírez, Gen. Rel. Grav. 34, $283(2002)$

29. S. Chandrasekhar, The Mathematical Theory of Black Holes (Oxford University Press, Oxford, 1983)

30. S.W. Hawking, Phys. Rev. D 46, 603 (1992)

31. M.S. Morris, K.S. Thorne, U. Yurtsever, Phys. Rev. Lett. 61, 148 (1988)

32. J. Friedmann, M.S. Morris, I.D. Novikov, F. Echeverria, G. Klinkhammer, K.S. Thorne, U. Yurtsever, Phys. Rev. D 42, 1915 (1990)

33. M. Novello, N.F. Svaiter, M.E.X. Guimarães, Mod. Phys. Lett. A 7, 381 (1992)

34. M. Novello, N.F. Svaiter, M.E.X. Guimarães, Gen. Rel. Grav. 25, 137 (1993)

35. M. Novello, M.C.M. da Silva, Phys. Rev. D 49, 825 (1994)

36. S.W. Hawking, The path integral approach to quantum gravity, in General Relativity, an Einstein Centenary Survey, ed. by S.W. Hawking, W. Israel (Cambridge University Press, Cambridge, 1979)

37. J.B. Hartle, S.W. Hawking, Phys. Rev. D 28, 2960 (1983) 